directly from the $X$-distribution. To do so, we replace $\mathbf{E} S_{[n / \log \log n]}^{+}$by an approximation thereto. With $K(\bullet)$ defined as in (2) and (3),

$$
0.673 \leqq \liminf _{n \rightarrow \infty} \frac{\mathbf{E} S_{n}^{+}}{K(n)} \leqq \limsup _{n \rightarrow \infty} \frac{\mathbf{E} S_{n}^{+}}{K(n)} \leqq 2 .
$$

(For a proof of (9), consult Klass [6].) Hence, letting $a_{n}=(\log \log n) K(n / \log \log n)$, our heuristics suggest that when the possibility of getting large $X_{j}^{+}$can be ignored, $L \equiv$ $\lim \sup _{n \rightarrow \infty} S_{n} / a_{n}$ should be both positive and finite. Indeed, as stated in (4), whenever $\mathbf{P}\left(X_{n}>a_{n}\right.$ i.o. $)=0$, we have $1 \leqq L \leqq 1.5$.

Thus, $a_{n}$ has been identified as the product of two quantities, $K(n / \log \log n)$ and $\log \log n$, each capable of interpretation. $K(n / \log \log n)$ relates to the size of a "piece of good luck" while $\log \log n$ relates to the maximum number of such pieces which may occur in succession infinitely often.

When $0<\mathbf{E} X^{2}=\sigma^{2}<\infty, K(n) \sim \sigma \sqrt{n}$ and so $\sqrt{2} a_{n} \sim \sigma(2 n \log \log n)^{1 / 2}=d_{n}$. On the other hand, if $X^{+}$is bounded above and $\mathbf{P}(X<-y)=\left(y \log ^{2} y\right)^{-1}$ for $y \geqq e$, then $K(n) \sim$ $n / \log n$ and $\mathbf{E} S_{n}^{+} \sim K(n)$. In this case, it follows that $a_{n} \sim \mathbf{E} S_{n}^{+}$. In the first case, $L \equiv$ $\lim \sup _{n \rightarrow \infty} S_{n} / a_{n}$ equals $\sqrt{2}$, while in the second case, $L=1$. These extreme cases illustrate how well the norming sequence $\left\{a_{n}\right\}$ adjusts to diverse situations, varying between $\mathbf{E} S_{n}^{+}$ and $d_{n}$.

Acknowledgment. I want to thank Shimon Friedman and $\mathrm{Ker}-\mathrm{Chau} \mathrm{Li}$, who examined an earlier draft of this manuscript, offering useful comments and suggestions.

Received by the editors December 9, 1982

\title{
REFERENCES
}

[1] A. KhINCHIN, Über dyadische Brüche, Math. Z., 18 (1923), pp. 109-116.

[2] A. KhINCHIN, Über einen Satz der wahrscheinlichkeitsrechnung, Fund. Math., 6 (1924), pp. 9-20.

[3] P. HARTMAN AND H. Wintner, On the law of the iterated logarithm, Amer. J. Math., 63 (1941), pp. 169-176.

[4] M. J. KLAss, Toward a universal law of the iterated logarithm, I; II, Z. Wahrsch. Verw. Geb., 36, 2 (1976), pp. 165-178; 39, 2 (1977), pp. 151-165.

[5] M. J. KLASS AND H. TEICHER, Iterated logarithm laws for asymmetric random variables barely with or without finite mean, Ann. Probab., 5 (1977), pp. 861-874.

[6] M. J. KLASS, Precision bounds for the relative error in the approximation of $\mathbf{E}\left|S_{n}\right|$ and extensions, Ann. Probab., 8 (1980), pp. 350-367.

[7] V. Strassen, An invariance principle for the law of the iterated logarithm, Z. Wahrsch. Verw. Geb., 3, 3 (1964), pp. 211-226.

\section{CENTERING OF SIGNED RANK STATISTICS WITH A CONTINUOUS SCORE-GENERATING FUNCTION}

\author{
M. L. PURI AND S. S. RALESCU
}

\begin{abstract}
Summary
For a continuous score generating function, Hájek [2] established the asymptotic normality of a simple linear rank statistic $S_{N}$ with natural parameters $\left(\mathbf{E} S_{N}, \operatorname{Var} S_{N}\right)$ as well as $\left(\mathbf{E} S_{N}, \sigma_{N}^{2}\right)$, where $\sigma_{N}^{2}$ is some constant. The permissibility of replacing $\mathbf{E} S_{N}$ by a simpler constant $\mu_{N}$ was shown by Hoeffding [4] under conditions slightly stronger than Hájek's.
\end{abstract}


Following Hájek's methods, Hušková [5] derived the asymptotic normality of a simple signed rank statistic $S_{N}^{+}$with parameters $\left(\mathbf{E} S_{N}^{+}\right.$, Var $\left.S_{N}^{+}\right)$as well as $\left(\mathbf{E} S_{N}^{2}, \sigma_{N}^{2}\right)$ and left open the problem of the replacement of $\mathbf{E} \boldsymbol{S}_{N}^{+}$by some simpler constant. In this note we close this problem of the replacement of $\mathbf{E} S_{N}^{+}$by a simpler constant $\mu_{N}^{+}$. The solution is a follow-up of Hoeffding [4]. We also provide a slight generalization with regard to the choice of scores.

\section{Introduction}

Let $X_{N i}, 1 \leqq i \leqq N, N \geqq 1$, be independent r.v.'s with continuous distribution functions $F_{N i}, 1 \leqq i \leqq N, N \geqq 1$, respectively. Hájek [2] studied the asymptotic distribution of the statistic $S_{N}=\sum_{i=1}^{N} c_{N i} a_{N}\left(R_{N i}\right)$, where the $c_{N i}, 1 \leqq i \leqq N$, are known constants, $R_{N i}, 1 \leqq i \leqq N$, are the ranks of $X_{N i}, 1 \leqq i \leqq N$, respectively, and $a_{N}(i)$ are the scores. Under suitable assumptions on the $c$ 's and the score generating function, Hájek [2] proved the asymptotic normality of $S_{N}$ with natural parameters $\left(\mathbf{E} S_{N}, \operatorname{Var} S_{N}\right)$ as well as $\left(\mathbf{E} S_{N}, \sigma_{N}^{2}\right)$, where $\sigma_{N}^{2}(>0)$ is some constant, and left open the problem of the replacement of $\mathbf{E} S_{N}$ by a simpler centering constant $\mu_{N}$. This problem was later investigated by Hoeffding [4], who showed that this replacement was permissible if one of Hájek's condition is slightly strengthened. Later, following the methods of Hájek [2], Hušková [5] derived the asymptotic normality of the signed rank statistic $S_{N}^{+}=\sum_{i=1}^{N} c_{N i} a_{N}\left(R_{N i}^{+}\right) \operatorname{sgn} X_{N i}$ (where the $c$ 's (as above) are known constants, $R_{N i}^{+}, 1 \leqq i \leqq N$, are the ranks of $\left|X_{N i}\right|, 1 \leqq i \leqq N$, among $\left.\left(\left|X_{N_{i}}\right|, 1 \leqq j \leqq N\right)\right)$ with the parameters $\left(\mathbf{E} S_{N}^{+}, \operatorname{Var} S_{N}^{+}\right)$as well as $\left(\mathbf{E} S_{N}^{+}, \sigma_{N}^{2}\right)$. Even though the statistic $S_{N}^{+}$is extensively used in both theroetical as well as applied problems, the problem of the replacement of $\mathbf{E} \boldsymbol{S}_{N}^{+}$by a simpler constant has not been formally considered. In this note we close this problem of the replacement of $\mathbf{E} S_{N}^{+}$by a simpler constant $\mu_{N}^{+}$. The solution is a straightforward follow-up of Hoeffding [4].

\section{Preliminaries}

We assume that the scores $a_{N}(i)$ are generated by a function $\varphi:(0,1) \rightarrow R$, which is expressible as a difference of two non-decreasing functions $\varphi_{1}$ and $\varphi_{2}$, that is $\varphi(t)=$ $\varphi_{1}(t)-\varphi_{2}(t), 0<t<1$.

The scores are obtained either by interpolation

$$
a_{N}(i)=\varphi(i /(N+1)),
$$$$
1 \leqq i \leqq N
$$

or by a procedure satisfying

$$
\sum_{i=1}^{N}\left|a_{N}(i)-\varphi(i /(N+1))\right|=o\left(N^{1 / 2}\right),
$$

or by

$$
a_{N}(i)=\mathbf{E} \varphi\left(U_{N}^{(i)}\right)
$$

$1 \leqq i \leqq N$,

where $U_{N}^{(i)}$ is the $i$ th order statistic in a random sample of size $N$ from the uniform distribution over $(0,1)$.

We also assume the nondegeneration of variance $\operatorname{Var} S_{N}^{+}$in the strict form:

$$
\liminf _{N \rightarrow \infty} \operatorname{Var}\left(S_{N}^{+}\right) /\left(N \max _{1 \leqq i \leqq N} c_{N i}^{2}\right)>0 .
$$

Alternatively, we may assume that (2.4) holds with $\operatorname{Var} S_{N}^{+}$replaced by some approximate variance $\sigma_{N}^{2}$ that is

Set

$$
\liminf _{N \rightarrow \infty} \sigma_{N}^{2} /\left(N \max _{1 \leqq i \leqq N} c_{N i}^{2}\right)>0
$$

$$
H_{N}(x)=N^{-1} \sum_{i=1}^{N} F_{N i}(x) \text { and } H_{N}^{*}(x)=N^{-1} \sum_{i=1}^{N} F_{N i}^{*}(x)
$$


where $F_{N i}^{*}(x)=\mathrm{P}\left[\left|X_{N i}\right| \leqq x\right], 1 \leqq i \leqq N$. For notational convenience, we suppress the subscript $N$ from $X_{N i}, F_{N i}, F_{N i}^{*}, R_{N i}^{+}$. etc.

We now quote Hušková's [5] main result transformed from her $\varepsilon$-form into a limit form.

Theorem (Hušková). Let $\varphi(t)=\varphi_{1}(t)-\varphi_{2}(t), 0<t<1$, where $\varphi_{1}(t)$ and $\varphi_{2}(t)$ are both nondecreasing, square integrable and absolutely continuous inside $(0,1)$. Consider $S_{N}^{+}$given in Section 1 with scores satisfying (2.1) or (2.3). Assume that (2.4) or (2.5) holds, where

$$
\sigma_{N}^{2}=\sum_{i=1}^{N} \operatorname{Var} Z_{i}
$$

$$
\begin{aligned}
Z_{i}=N^{-1} \sum_{j=1}^{N} c_{j} \int_{-\infty}^{\infty} \operatorname{sgn} x\left[u\left(|x|-\left|X_{i}\right|\right)-F_{i}^{*}(|x|)\right] \varphi^{\prime}\left(H^{*}(|x|)\right) d F_{j}(x) & \\
& +c_{i}\left[\operatorname{sgn} X_{i} \varphi\left(H^{*}\left(\left|X_{i}\right|\right)\right)-\mathbf{E}\left(\operatorname{sgn} X_{i} \varphi\left(H^{*}\left(\left|X_{i}\right|\right)\right)\right)\right], \quad 1 \leqq i \leqq N .
\end{aligned}
$$

Then $S_{N}^{+}$is asymptotically, normal with parameters $\left(\mathbf{E} S_{N}^{+}, \operatorname{Var} S_{N}^{+}\right)$as well as with parameters $\left(\mathbf{E} S_{N}^{+}, \sigma_{N}^{2}\right)$.

\section{Main Result}

In this section we show that if the condition of square integrability of $\varphi_{1}$ and $\varphi_{2}$ is slightly strengthened, then Hušková's theorem remains true with $\mathbf{E} S_{N}^{+}$replaced by

$$
\mu_{N}^{+}=\sum_{i=1}^{N} c_{i} \int_{-\infty}^{\infty} \operatorname{sgn} x \varphi\left(H^{*}(|x|)\right) d F_{i}(x) .
$$

This problem is of practical interest, since $\mu_{N}^{+}$is quite simple and easier to manipulate in asymptotic situations.

Explicitly, we prove the following theorem.

Theorem 3.1. Consider the statistic $S_{N}^{+}$with $\varphi(t)=\varphi_{1}(t)-\varphi_{2}(t)$, where $\varphi_{1}$ and $\varphi_{2}$ are nondecreasing, absolutely continuous inside $(0,1)$ and satisfy the Hoeffding condition, viz.

$$
\int_{0}^{1} t^{1 / 2}(1-t)^{1 / 2} d \varphi_{i}(t)<\infty, \quad i=1,2 .
$$

Assume that the scores $a_{N}(i)$ are given by (2.1) or more generally by (2.2). Then either (2.4) or (2.5) with $\sigma_{N}^{2}$ defined by (2.6) implies the asymptotic normality of $S_{N}^{+}$with parameters $\left(\mu_{N}^{+}, \operatorname{Var} S_{N}^{+}\right)$as well as with parameters $\left(\mu_{N}^{+}, \sigma_{N}^{2}\right)$. Also, the above assertion remains true if the scores are generated by (2.3).

REMARK. If $\varphi$ is nondecreasing, then denoting

$$
J(\varphi)=\int_{0}^{1} t^{1 / 2}(1-t)^{1 / 2} d \varphi(t)
$$

it is shown in Hoeffding [4] that the condition $J(\varphi)<\infty$ implies the square integrability of $\varphi$, and the condition $\int_{0}^{1} \varphi^{2}(t)\{\log (1+|\varphi(t)|)\}^{1+\delta} d t<\infty$ for some $\delta>0$ implies $J(\varphi)<\infty$. In this sense the condition (3.2) is not much stronger than square integrability.

For the rest of this section, $C_{1}, C_{2}, \cdots$ will denote positive numerical constants numbered in order of appearance. Their possible dependence on other elements (functions, parameters, etc.) will also be emphasized.

The proof of Theorem 2.1 uses the techniques of Sections 3 and 4 of Hoeffding [4] and depends on the following lemmas.

Lemma 2.1. There exists a numerical constant $C_{1}>0$ such that if $\varphi$ is nondecreasing, then

$$
\sum_{i=1}^{N}\left|\mathbf{E}\left(\operatorname{sgn} X_{i} \varphi\left(\frac{R_{i}^{+}}{N+1}\right)\right)-\int_{-\infty}^{\infty} \operatorname{sgn} x \varphi\left(H^{*}(|x|)\right) d F_{i}(x)\right| \leqq C_{1} N^{1 / 2} J(\varphi),
$$

where $J(\varphi)$ is defined by (3.3). 
Proof. Set $\quad V(x)=\sum_{k=1}^{N} u\left(x-\left|X_{k}\right|\right), \quad V_{i}(x)=\left[1-u\left(x-\left|X_{i}\right|\right)\right]+V(x), \quad 1 \leqq i \leqq N$. Then, by conditioning, we have

$$
\begin{array}{ll}
\left|\mathbf{E}\left(\operatorname{sgn} X_{i} \varphi\left(\frac{R_{i}^{+}}{N+1}\right)\right)-\int_{-\infty}^{\infty} \operatorname{sgn} x \varphi\left(H^{*}(|x|)\right) d F_{i}(x)\right| & \\
\quad \leqq \int_{-\infty}^{\infty} \mathbf{E}\left|\varphi\left(\frac{V_{i}(|x|)}{N+1}\right)-\varphi\left(H^{*}(|x|)\right)\right| d F_{i}(x), & 1 \leqq i \leqq N .
\end{array}
$$

Now, by considerations as used in the derivation of (3.7) in Hoeffding [4], we obtain

$$
\begin{aligned}
& \sum_{i=1}^{N} \int_{-\infty}^{\infty} \mathbf{E}\left|\varphi\left(\frac{V_{i}(|x|)}{N+1}\right)-\varphi\left(H^{*}(|x|)\right)\right| d F_{i}(x) \\
& \quad \leqq 2 N \int_{0}^{\infty} \mathbf{E}\left|g(V(x))-\varphi\left(H^{*}(x)\right)\right| d H^{*}(x)+\left\{\varphi\left(\frac{N}{N+1}\right)-\varphi\left(\frac{1}{N+1}\right)\right\}
\end{aligned}
$$

where

Furthermore,

$$
g(i)=\min \left\{\varphi\left(\frac{i+1}{N+1}\right), \varphi\left(\frac{N}{N+1}\right)\right\}, \quad 0 \leqq i \leqq N
$$

$$
\begin{aligned}
\int_{0}^{\infty} \mathbf{E}\left|g(V(x))-\varphi\left(H^{*}(x)\right)\right| d H^{*}(x) \\
\quad \leqq \int_{0}^{\infty} \mathbf{E}\left|g(V(x))-g\left(\left[N H^{*}(x)\right]\right)\right| d H^{*}(x)+\int_{0}^{1}|g([N t])-\varphi(t)| d t
\end{aligned}
$$

$([\cdot]=$ integer part).

Consider now a random variable $W_{N}(p) \sim B(N, p)$. Then, following Hoeffding [3] it is easily shown that the first integral in the right-hand side of (3.7) is bounded from above by $\int_{0}^{1} \mathbf{E}\left|g\left(W_{N}(t)\right)-g([N t])\right| d t \leqq 4 N^{-1 / 2} J(\varphi)$.

This, together with (3.6) and (3.7) imply (via Hoeffding's [4] relations (3.18) through (3.20)) that

$$
\begin{aligned}
& \sum_{i=1}^{N} \int_{-\infty}^{\infty} \mathbf{E}\left|\varphi\left(\frac{V_{i}(|x|)}{N+1}\right)-\varphi\left(H^{*}(|x|)\right)\right| d F_{i}(x) \\
& \quad \leqq 8 N^{1 / 2} J(\varphi)+3(N+1)\left\{\int_{N /(N+1)}^{1} \varphi(t) d t-\int_{0}^{1 /(N+1)} \varphi(t) d t\right\} \\
& \quad \leqq C_{1} N^{1 / 2} J(\varphi)
\end{aligned}
$$

for some $C_{1}>0$. The proof follows from (3.5) and (3.8)

Lemma 3.2. If $\varphi:(0,1) \rightarrow R$ has a bounded second derivative, then there exists a constant $C_{2}(\varphi)>0$ such that, for all $i=1, \cdots, N$,

$$
\left|\mathbf{E}\left(\operatorname{sgn} X_{i} \varphi\left(R_{i}^{+} /(N+1)\right)\right)-\mathbf{E}\left(\operatorname{sgn} X_{i} \varphi\left(H^{*}\left(\left|X_{i}\right|\right)\right)\right)\right| \leqq C_{2}(\varphi) / N .
$$

Proof. The proof follows by using conditioning arguments, Taylor expansions and routine computations.

In the sequel, we introduce the symbols $S_{\varphi}^{+}, \mu_{\varphi}^{+}, Z_{i}(\varphi)$ and $\sigma_{\varphi}^{2}$ for the statistic $S_{N}^{+}$, the constant (3.1) and the random variables and the constant (2.6) if the scores are given by $a_{N}(i)=\varphi((i / N+1))$ to exhibit dependence on $\varphi$, leaving $S_{N}^{+}$for the general case of the scores given by $(2.2)$. Then we have

Lemma 3.3.

$$
\mathbf{E}\left(S_{N}^{+}-S_{\varphi}^{+}\right)^{2} \leqq\left(\max _{1 \leqq i \leqq N} c_{i}^{2}\right)\left(\sum_{i=1}^{N}\left|a_{N}(i)-\varphi\left(\frac{i}{N+1}\right)\right|\right)^{2} .
$$


Proof. By definition

$$
S_{N}^{+}-S_{\varphi}^{+}=\sum_{i=1}^{N} c_{i}\left\{\operatorname{sgn} X_{i}\left[a_{N}\left(R_{i}^{+}\right)-\varphi\left(\frac{R_{i}^{+}}{N+1}\right)\right]\right\}
$$

which implies

$$
\left|S_{N}^{+}-S_{\varphi}^{+}\right| \leqq\left(\max _{1 \leqq i \leqq N}\left|c_{i}\right|\right) \sum_{i=1}^{N}\left|a_{N}(i)-\varphi\left(\frac{i}{N+1}\right)\right|
$$

with probability one. The result follows.

Lemma 3.4. For scores given by $a_{N}(i)=\varphi(i /(N+1)),(2.4)$ holds if and only if (2.5) holds with $\sigma^{2}=\sigma_{\varphi}^{2}$ given by (2.6). In this case,

$$
\lim _{N \rightarrow \infty} \operatorname{Var} S_{\varphi}^{+} / \sigma^{2}=1 .
$$

Proof. With $\alpha>0$ to be specified later, we decompose $\varphi$ (cf. Hájek [2], Lemma 5.1) as $\varphi=\psi+\varphi_{(1)}-\varphi_{(2)}$, where $\psi$ is a polynomial, $\varphi_{(1)}$ and $\varphi_{(2)}$ are nondecreasing and

$$
\int_{0}^{1} \varphi_{(1)}^{2}(t) d t+\int_{0}^{1} \varphi_{(2)}^{2}(t) d t<\alpha .
$$

By Lemma 5 of Hušková [5], there exists a constant $C_{3}>0$ such that

$$
\sigma_{\varphi(k)}^{2}=\sum_{i=1}^{N} \operatorname{Var} Z_{i}\left(\varphi_{(k)}\right) \leqq C_{3} N\left(\max _{1 \leqq i \leqq N} c_{i}^{2}\right) \int_{0}^{1} \varphi_{(k)}^{2}(t) d t, \quad k=1,2,
$$

which together with the decomposition (3.12) implies

$$
\left|\sigma_{\varphi}-\sigma_{\psi}\right| \leqq \sigma_{\varphi_{(1)}}+\sigma_{\varphi_{(2)}} \leqq\left(2 C_{3}\right)^{1 / 2}\left(\max _{1 \leqq i \leqq N}\left|c_{i}\right|\right) N^{1 / 2} \alpha^{1 / 2} .
$$

From Lemma 2 of Hušková [5] we find that

$$
\left|\sigma_{\psi}-\left(\operatorname{Var} S_{\psi}^{+}\right)^{1 / 2}\right| \leqq C_{4}(\psi) \max _{1 \leqq i \leqq N}\left|c_{i}\right|, \quad \text { where } C_{4}(\psi)>0 .
$$

Finally, using Theorem 4 of Hušková [5] and (3.11) we obtain

$$
\left|\left(\operatorname{Var} S_{\varphi}^{+}\right)^{1 / 2}-\left(\operatorname{Var} S_{\psi}^{+}\right)^{1 / 2}\right| \leqq(160)^{1 / 2}\left(\max _{1 \leqq i \leqq N}\left|c_{i}\right|\right) N^{1 / 2} \alpha^{1 / 2} .
$$

Now suppose that (2.4) holds. Let $\gamma>0$ be such that

$$
\operatorname{Var} S_{\varphi}^{+} \geqq \gamma N \max _{1 \geqq i \geqq N} c_{i}^{2}, \quad N \geqq 1 .
$$
such that

Then, using (3.13)-(3.16), there exists a constant $C_{5}(\gamma)>0$, depending on $\gamma$ only

$$
\left|\left(\sigma_{\varphi}^{2} / \operatorname{Var} S_{\varphi}^{+}\right)^{1 / 2}-1\right| \leqq C_{5}(\gamma) \alpha^{1 / 2}+\left(C_{4}(\psi) / \gamma\right)^{1 / 2} N^{-1 / 2}
$$

Let $\varepsilon>0$ be given. Choose $\alpha$ such that $C_{5}(\gamma) \alpha^{1 / 2}=\varepsilon / 2$. This choice fixes $C_{4}(\psi)$. Then from (3.17) we clearly obtain (3.11). Consequently, (2.5) holds true. Similarly, (2.5) implies (3.17) and thus (2.4). The proof follows.

Lemma 3.5. Let the scores be given by (2.2). Then

$$
\liminf _{N \rightarrow \infty} \operatorname{Var}\left(S_{N}^{+}\right) /\left(N \max _{1 \leqq i \cong N} c_{i}^{2}\right)>0 \Leftrightarrow \liminf _{N \rightarrow \infty} \operatorname{Var}\left(S_{\varphi}^{+}\right) /\left(N \max _{1 \leqq i \leqq N} c_{i}^{2}\right)>0 .
$$

In this case, $\lim _{N \rightarrow \infty} \operatorname{Var}\left(S_{\varphi}^{+}\right) / \operatorname{Var}\left(S_{N}^{+}\right)=1$. 
ProOF. The proof follows by using

$$
\left\{\left(\frac{\operatorname{Var} U_{1}}{\operatorname{Var} U_{2}}\right)^{1 / 2}-1\right\}^{2} \leqq \frac{\operatorname{Var}\left(U_{1}-U_{2}\right)}{\operatorname{Var} U_{2}}
$$

together with Lemma 3.3.

ProOF OF THEOREM 3.1. Assume that the scores are given by (2.1). Since from Theorem 6 of Hušková [5], $\left(S_{\varphi}^{+}-\mathbf{E} S_{\varphi}^{+}\right) /\left(\operatorname{Var} S_{\varphi}^{+}\right)^{1 / 2} \stackrel{\mathscr{C}}{\rightarrow} N(0,1)$, the asymptotic normality of $S_{\varphi}^{+}$with parameters $\left(\mu_{\varphi}^{+}, \operatorname{Var} S_{\varphi}^{+}\right)$will follow if we show that

$$
\lim _{N \rightarrow \infty}\left(\mathbf{E} S_{\varphi}^{+}-\mu_{\varphi}^{+}\right) /\left(\operatorname{Var} S_{\varphi}^{+}\right)^{1 / 2}=0 .
$$

To this end, with $\alpha>0$ to be specified later, we decompose $\varphi$ as in (Hoeffding [4], Lemma $1)$, viz. $\varphi=\psi+\varphi_{(1)}-\varphi_{(2)}$, where $\psi$ is a polynomial, $\varphi_{(1)}$ and $\varphi_{(2)}$ are nondecreasing and

$$
J\left(\varphi_{(1)}\right)+J\left(\varphi_{(2)}\right)<\alpha .
$$

Now, for any $\varphi$, it is readily seen that

$$
\left|\mathbf{E} S_{\varphi}^{+}-\mu_{\varphi}^{+}\right| \leqq\left(\max _{1 \leqq i \leqq N}\left|c_{i}\right|\right) \sum_{i=1}^{N}\left|\mathbf{E}\left(\operatorname{sgn} X_{i} \varphi\left(\frac{R_{i}^{+}}{N+1}\right)\right)-\int_{-\infty}^{\infty} \operatorname{sgn} x \varphi\left(H^{*}(|x|)\right) d F_{i}(x)\right| .
$$

Applying Lemma 3.2 and (3.20) with $\varphi=\psi$ we obtain

$$
\left|\mathbf{E} S_{\psi}^{+}-\mu_{\psi}^{+}\right| \leqq C_{2}(\psi) \max _{1 \leqq i \leqq N}\left|c_{i}\right| \text {. }
$$

Also from Lemma 3.1, (3.19) and (3.20) with $\varphi=\varphi_{(k)}, k=1,2$, we have

$$
\sum_{k=1}^{2}\left|\mathbf{E} S_{\varphi(k)}^{+}-\mu_{\varphi(k)}^{+}\right| \leqq C_{1} N^{1 / 2} \alpha \max _{1 \leqq i \leqq N}\left|c_{i}\right| \text {. }
$$

Let $\gamma>0$ be such that (2.16) holds. Then from (3.16), (3.20) and (3.21) we get

$$
\left|\mathbf{E} S_{\varphi}^{+}-\mu_{\varphi}^{+}\right| /\left(\operatorname{Var} S_{\varphi}^{+}\right)^{1 / 2} \leqq C_{2}(\psi) \alpha^{-1 / 2} N^{-1 / 2}+C_{1} \gamma^{-1 / 2} \alpha .
$$

Now, given $\varepsilon>0$, choose $\alpha>0$ in the decomposition (3.20) such that $C_{1} \gamma^{-1 / 2} \alpha=\varepsilon / 2$. This choice fixes $\psi$ and therefore $C_{2}(\psi)$. Finally, choose $N_{\varepsilon}$ such that $C_{2}(\psi) \gamma^{-1 / 2} N^{-1 / 2}<$ $\varepsilon / 2$ for $N \geqq N_{\varepsilon}$. Then (3.22) implies (3.18) as was to be shown. Also Lemma 2.4 implies the asymptotic normality of $S_{\varphi}^{+}$with parameters $\left(\mu_{\varphi}^{+}, \sigma_{\varphi}^{2}\right)$.

Suppose now that the scores are given by (2.2). Then using Lemma 3.5, (3.18) and the fact that $\left(S_{N}^{+}-\mathbf{E} S_{N}^{+}-S_{\varphi}^{+}+\mathbf{E} S_{\varphi}^{+}\right) /\left(\operatorname{Var} S_{N}^{+}\right)^{1 / 2} \stackrel{L^{2}}{\rightarrow} 0$ (which follows from Lemma 3.3) we obtain the asymptotic normality of $S_{N}^{+}$with natural parameters $\left(\mathbf{E} S_{N}^{+}, \operatorname{Var} S_{N}^{+}\right)$. Finally, proceeding as before we obtain the asymptotic normality of $S_{N}^{+}$with parameters $\left(\mu_{N}^{+}, \operatorname{Var} S_{N}^{+}\right),\left(\mathbf{E} S_{N}^{+}, \sigma_{N}^{2}\right)$ and $\left(\mu_{N}^{+}, \sigma_{N}^{2}\right)$.

The case of the "exact" scores $(2.3)$, i.e., $a_{N}(i)=\mathbf{E} \varphi\left(U_{N}^{(i)}\right)$, can be treated similarly as in Hoeffding $([4])$, p. 62$)$ with the help of the inequality

$$
\begin{aligned}
\left|\mathbf{E} S_{\varphi}^{+}-\mu_{\varphi}^{+}\right| \leqq & \left(\max _{1 \leqq i \leqq N}\left|c_{i}\right|\right)\left\{\sum_{i=1}^{N}\left|\mathbf{E} \varphi\left(U_{N}^{(i)}\right)-\varphi\left(\frac{i}{N+1}\right)\right|\right. \\
& \left.+\sum_{i=1}^{N}\left|\mathbf{E}\left(\operatorname{sgn} X_{i} \varphi\left(\frac{R_{i}^{+}}{N+1}\right)\right)-\int_{-\infty}^{\infty} \operatorname{sgn} x \varphi\left(H^{*}(|x|)\right) d F_{i}(x)\right|\right\}
\end{aligned}
$$

holding for any $\varphi$. 


\title{
REFERENCES
}

[1] H. Chernoff AND I. R. SAvage, Asymptotic normality and efficiency of certain non-parametric test statistics, Ann. Math. Statist. 29 (1958), pp. 972-994.

[2] J. HÁJEK, Asumptotic normality of simple linear rank statistics under alternatives, Ann. Math. Statist., 39 (1968), pp. 325-346.

[3] W. HoEfFDing, On the distribution of the number of successes in independent trials, Ann. Math. Statist., 27 (1956), pp. 713-721.

[4] W. HoEFFDING, On the centering of a simple linear rank statistic, Ann. Statist., 1 (1973), pp. 56-66.

[5] M. HuŠKovà. Asymptotic distribution of simple linear rank statistics for testing symmetry, $\mathrm{Z}$. Waharsch. Verw. Geb., 14 (1970), pp. 308-322.

\section{THE SINGLE JUMP PROCESS WITH SOME STATISTICAL APPLICATIONS}

\author{
A. AL-HUSSAINI AND R. J. ELLIOTT
}

\section{Introduction}

Martingales associated with the single jump process were discussed by Chou and Meyer [1], Davis [2] and Elliott [3]. In this paper we indicate how some results of these papers extend to the situation where the time parameter runs through the real numbers and then apply them to a real-valued random variable. In particular, a representation for the empirical distribution is obtained in Section 3. A martingale proof of the GlivenkoCantelli theorem is then derived. The Glivenko-Cantelli theorem states that the empirical distribution function of a random variable converges uniformly on the whole line to the distribution function. It has important applications in mathematical statistics, and the usual proofs (see, for example, [4]), are measure-theoretic. Finally, following a suggestion of Mark Davis, the functional central limit theorem of Liptser and Shiryaev [6] is applied in Section 5 to deduce a convergence of the empirical distribution to the Brownian bridge.

The second author wishes to thank Professor Al-Hussaini for arranging his visit to Edmonton in the summer of 1981, when work on this paper commenced.

\section{Random Variables and Martingales}

In this section we indicate how some results of Chou and Meyer [1], Davis [2], and Elliott [3], concerning the single jump process, extend to processes associated with a real-valued random variable. The only new feature compared with the above references is that the "times" parameter here belongs to $[-\infty, \infty]$ rather than $[0, \infty]$, so proofs will not be given.

Consider a random variable $X$ with values in $[-\infty, \infty]$. As the basic probability space we can, therefore, take $\Omega=[-\infty, \infty]$ with the Borel field $\mathscr{B}$. Suppose

$$
G_{t}=\mathbf{P}(X \leqq t), \quad t \in[-\infty, \infty],
$$

is the distribution function of $X$. We shall also assume that $G_{-\infty}=F_{\infty}=0$, where $F_{t}=1-G_{t}$. The Stieltjes measure $d G_{t}$ gives rise to a probability measure on $(\Omega, \mathscr{B})$. Rather than $G_{t}$ it is often more convenient in the calculations below to use

$$
F_{t}=\mathbf{P}(X>t)=t-G_{t} .
$$

Consider the indicator process generated by searching along the real line in the positive direction for the value $X$ of the random variable, i.e., the process $p_{t}=I_{t \geqq X}$. Write 
Reproduced with permission of the copyright owner. Further reproduction prohibited without permission. 